\title{
La Pensée Critique en Éducation Physique et à la Santé (EPS) : Sens et Utilité?
}

\section{Kpazaï Georges, PhD}

École des sciences de l'activité physique (ÉSAP), Faculté de la santé,

Université Laurentienne, Sudbury (Ontario), Canada

\section{Ben Jomâa, Hejer, PhD}

Institut Supérieur du Sport et de l'Éducation Physique (ISSEP) de Ksar-Said,

Université de La Manouba, Tunisie

\section{Mandoumou Paulin, PhD}

Institut Supérieur d'Éducation Physique et Sportive (ISEPS),

Université Marien Ngouabi (UMNG), République du Congo

\section{Hariti Hakim, PhD}

Institut de l'Éducation Physique et Sportive (IÉPS),

Université d'Alger 3, Algérie

\section{Attiklémé Kossivi, PhD}

Institut National de la Jeunesse, de l'Éducation Physique et du Sport (INJEPS), Université d'Abomey-Calavi (UAC), République du Bénin

Doi: 10.19044/esj.2019.v15n15p86 URL:http://dx.doi.org/10.19044/esj.2019.v15n15p86

\section{Résumé}

Quel est le portrait de la pensée critique dans l'agir professionnel des enseignants d'EPS ? Quel sens et quelle utilité a cette forme de pensée dans une classe d'enseignement de l'EPS ? Basée sur la conception lipmanienne de la pensée critique (Lipman, 1995, 2006), la présente étude tente d'émettre des éléments de compréhension de la signification et de la portée de la pensée critique lorsqu'elle est exprimée dans la pratique enseignante des éducateurs physiques scolaires. Pour ce faire, neuf enseignements de classes d'EPS délivrés par trois enseignants du secondaire ont fait l'objet d'observations et d'explicitations via la technique de rappel stimulé. Les résultats révèlent que la pensée critique déployée lors du processus enseignement-apprentissage, bien qu'elle ait des expressions variables chez les enseignants, est essentielle à la pertinence de l'acte éducatif de ceux-ci. Elle favorise, dans la plupart des cas, l'apprentissage et l'éducation des élèves.

Mots-clés : Pensée critique, éducation physique et santé, enseignants en exercice 


\title{
Critical Thinking in Health and Physical Education (HPE): Meaning and Usefulness?
}

\section{Kpazaï Georges, PhD}

École des sciences de l'activité physique (ÉSAP), Faculté de la santé,

Université Laurentienne, Sudbury (Ontario), Canada

\section{Ben Jomâa, Hejer, PhD}

Institut Supérieur du Sport et de l'Éducation Physique (ISSEP) de Ksar-Said,

Université de La Manouba, Tunisie

\section{Mandoumou Paulin, PhD}

Institut Supérieur d'Éducation Physique et Sportive (ISEPS),

Université Marien Ngouabi (UMNG), République du Congo

\section{Hariti Hakim, PhD}

Institut de l'Éducation Physique et Sportive (IÉPS),

Université d'Alger 3, Algérie

\section{Attiklémé Kossivi, PhD}

Institut National de la Jeunesse, de l'Éducation Physique et du Sport (INJEPS), Université d'Abomey-Calavi (UAC), République du Bénin

\begin{abstract}
What is the portrait of Critical Thinking in the professional action of HPE Teachers? What are the meaning and usefulness of this form of thinking in a HPE setting? Based on Lipman's conception of Critical Thinking (Lipman, 1995, 2006), this study attempts to provide some understanding of the meaning and scope of Critical Thinking when expressed in the Teaching practice of HPE Teachers. To do this, nine HPE classes taught by three secondary school teachers were subject to observations and explanations via the stimulated recall technique. The results reveal that Critical Thinking in the teaching-learning process, although it has variable expressions among teachers, is essential to the relevance of Teachers' educational act. It promotes, in most cases, student learning and education.
\end{abstract}

Keywords: Critical Thinking, Health and Physical Education, In-service Teachers 


\section{Introduction}

Bien qu'il soit vrai que la thématique de la « pensée critique » ne soit pas récente dans la littérature en éducation, un certain regain d'intérêt à son endroit apparaît aujourd'hui dans ce domaine depuis plus de deux décennies. Par exemple, dans la plupart des curricula, le développement de la pensée critique des apprenants est présenté comme un objectif éducationnel prioritaire (Ministère de l'Éducation de la Colombie Britannique, 2002; Ministère de l'Éducation de la Nouvelle Zélande, 2005 ; Ministère de l'Éducation, des Loisirs et du Sport du Québec, 2001a, 2001b, 2003; Ministère de l'Éducation de l'Ontario, 2015; Ministère de l'Enseignement Primaire et Secondaire du Bénin, 2006). Or, si les institutions éducatives souhaitent l'acquisition et le développement de la pensée critique des apprenants, plusieurs conditions, selon la recherche sur la pensée critique, doivent être réunies. D'une part, les professionnels du vaste monde de l'éducation doivent s'entendre exactement sur la nature et l'essence réelles du concept de «pensée critique » en enseignement et, d'autre part, s'assurer que les enseignants eux-mêmes déploient une forme de pensée dite «critique » lors du processus enseignement-apprentissage (Daniel, 1998a; Ennis, 1991; Knight, 1992).

Pour la première condition susmentionnée, la plupart des savants du domaine de la pensée critique font observer qu'il existe une multitude de définitions. Toutefois, non seulement un petit nombre de ces définitions sont opérationnalisables (Kpazaï, 2018, 2015), mais toutes ces définitions de la pensée critique n'apportent pas encore un éclairage parfait sur la pensée critique (Doherty, 2009; Kpazaï, Daniel et Attiklémé, 2015, 2011; McBride et Xiang, 2004). Dès cet instant, on peut se poser la question à savoir : comment développer convenablement et, conséquemment, comment évaluer correctement cette forme de pensée chez les apprenants?

Concernant la deuxième condition, plusieurs chercheurs en éducation physique ont mentionné le rôle crucial des éducateurs physiques dans l'acquisition et le développement de la pensée critique des élèves dans le domaine psychomoteur (Bennett et Green, 2001; Cleland, Helion et Fry, 1999; Daniel, 2001; Daniel et Bergman-Drewe, 1998; Kpazaï, 2013; McBride, 1999; McBride et Xiang, 2004). Pour la plupart de ces chercheurs, l'enseignant d'éducation physique, par l'adoption d'attitudes et d'aptitudes à la pensée critique, peut non seulement favoriser la qualité de l'apprentissage des élèves, mais aussi permettre l'usage de la pensée critique par ces derniers. Toutefois, pour que les enseignants assument cette pleine responsabilité, Gaskins (1994) souligne qu'il est indispensable qu'ils comprennent la cognition, prennent conscience de la variété des stratégies cognitives et soient capables d'analyser les composantes de ces stratégies en vue de leur enseignement. Pour Knight (1992), si les enseignants désirent développer 
adéquatement la pensée critique des élèves, ils doivent satisfaire trois exigences : 1) maîtriser la discipline, 2) être eux-mêmes dotés de plusieurs capacités et attitudes de la pensée critique et 3) savoir comment enseigner à penser. Quant à Newman (1990), les enseignants doivent être de «bons penseurs critiques » afin de développer la pensée critique des apprenants. Enfin Daniel (1998b) fait la remarque suivante à l'endroit des enseignants :

«Dans le but d'aider les enfants à bien penser, à devenir autonomes, critiques et ouverts, demandez-vous « est-ce que je suis moi-même une personne qui se questionne constamment, qui cherche toujours des réponses de plus en plus plausibles, qui est intéressé à dialoguer et à découvrir? » (Daniel, 1998b, p.99)

Ainsi, la question de la pensée critique concerne aussi bien les élèves que les enseignants. Dès lors, elle devient donc une préoccupation à la fois de l'apprentissage et de l'enseignement. Elle a donc trait à la problématique de l'enseignement et plus spécifiquement à la réflexivité critique en enseignement. Cette réflexivité critique est assimilable à la pensée réflexive au sens de Dewey (1933).

Par ailleurs, pour certains chercheurs, la recherche actuelle sur la pensée critique chez les enseignantes et les enseignants est, pour une bonne partie, imposée par la recherche des nouveaux indices relatifs à la qualité de l'enseignement et de l'apprentissage, surtout à celle de l'éducation. Dans cette perspective, la tendance actuelle est la prise en compte du processus cognitif des enseignants (Durand, 1998; Howe, 2004; Kpazaï, 2014). Un courant de recherche pédagogique, intitulé « la pensée des enseignants », postule à cet effet que les comportements observés en classe chez les enseignants sont largement influencés par ce qu'ils pensent. Leurs habiletés cognitives sont donc conçues comme des éléments explicatifs de leurs comportements car elles sont censées influencer ces derniers.

Déjà en 1970, Taylor faisait remarquer que même ces professionnels technicistes de l'enseignement faisaient des choix lors de leur planification pédagogique. Il précise même que pendant la phase d'interaction, les enseignants pensent, choisissent, s'adaptent ou modifient leurs comportements. Même si l'objet de cette réflexion ne se limitait qu'au contexte d'enseignement-apprentissage et à ses buts poursuivis, Taylor fait penser à l'existence d'un processus cognitif sous-jacent aux comportements et actes des enseignants. Shavelson (1973) affirme, dans cette ligne de pensée, que tout acte d'enseignement est le résultat d'une décision, soit consciente ou inconsciente de l'enseignant après qu'il ait opéré un traitement complet de l'information disponible. On peut donc affirmer que l'implication du processus cognitif de l'enseignant lors du processus enseignement- 
apprentissage est essentielle à l'à-propos de l'acte pédagogique et au développement professionnel. La pensée réflexive des enseignants est fondamentale à leur développement professionnel. À ce propos, Sebren (1995) écrit :

«Si les changements de qualité qui interviennent lorsque les enseignantes et les enseignants acquièrent leur expertise sont caractérisés par des changements dans la nature des relations de leurs structures cognitives, et si ces relations sont considérées comme le point culminant de la réflexion, alors la réflexion doit jouer un rôle fondamental dans le développement des enseignantes et des enseignants ». (p. 264)

Smyth (1984) partage le même point de vue lorsqu'il précise qu'un tel processus permet à l'enseignant et à l'enseignante d'évaluer les anachronismes qui ont pu s'installer au fil des ans de sa pratique pédagogique.

«Quand les enseignantes et les enseignants adoptent, eux-mêmes, une attitude réflexive envers leur enseignement, ils s'engagent dans un processus de problématisation ou de questionnement des aspects de l'enseignement pris généralement comme acquis ». (p. 60)

Selon Tsangaridou et Siedentop (1995), la mise en place d'une pédagogie basée sur la réflexion critique permettra : 1) une réflexion sur les conditions sociales dans lesquelles les pratiques éducatives ont lieu, 2) une réflexion dans une perspective démocratique et émancipatrice dans le sens où elle est centrée sur les inégalités qui prennent place dans l'enseignement et dans l'école et 3) une réflexion qui tente de développer une conscience sociale par le biais de la mise en place d'une «communauté d'apprentissage ».

Bref, si la littérature scientifique dans le domaine de la pensée critique contient des données manifestes pour répondre à la question «comment enseigner pour développer la pensée critique des élèves et étudiants », il y a cependant peu de réponses issues de recherches empiriques à l'égard de la question «est-ce que les enseignants d'EPS font preuve de pensée critique en enseignement? "Si oui, quels sont le sens et l'utilité de la pensée critique ainsi manifestée?

\section{Cadre conceptuel}

Le cadre conceptuel est essentiellement constitué de la conception de la pensée critique mise de l'avant par Mathiew Lipman (1995, 2006). Cette conception s'inspire de celle de J. Dewey (1933) relative à la pensée réflexive. Pour Daniel et Bergman-Drewe (1998), la philosophie dialogique mise de l'avant, dans les travaux de Lipman (1995, 2006), non seulement prend cours 
dans une communauté de recherche, mais vise essentiellement au développement des habiletés et des dispositions reliées à la pensée critique. De plus, pour plusieurs chercheurs du domaine, la conception lipmanienne de la pensée critique a l'avantage de fournir des indicateurs de la pensée critique (Lafortune et Robertson, 2004; Roy, 2005). Ainsi, selon Lipman (1995, 2006), en plus du fait que la pensée critique doit aboutir à un bon jugement, elle doit répondre à trois caractéristiques : 1) être basée sur des critères, 2) être sensible au contexte et 3 ) être autocorrectrice.

\section{Caractéristique 1 : La présence de critères}

Pour Lipman (1995), la pensée critique est « un mode de pensée qui à la fois utilise des critères et existe par le recours à des critères » (p.146). Sans eux, la pensée critique serait molle, sans forme, arbitraire, laissée au hasard et non structurée. Par contre, leur présence fait de la pensée critique, une pensée fondée, structurée et solide, car défendable et convaincante. Les critères lui sont donc essentiels, car ils lui donnent du poids. En fait, les critères sont présentés comme des règles ou des principes qui permettent à un individu de poser un jugement de façon adéquate.

\section{Caractéristique 2 : La présence d'une sensibilité au contexte}

La pensée critique est sensible au contexte en ce qu'elle reconnaît la spécificité de chaque cas, de chaque contexte et elle a un souci d'y répondre de manière appropriée et viable. La caractéristique « sensibilité au contexte » impose à la pensée critique d'être flexible dans l'application des principes à la pratique, de refuser d'attribuer le caractère normatif aux principes et aux faits et de s'engager dans une démarche propre à chaque contexte.

\section{Caractéristique 3 : La présence d'une autocorrection}

La pensée critique est autocorrectrice dans le sens où elle invite tout individu à partir à la découverte de ses propres faiblesses et erreurs, à être sensible à ses propres limites et être enclin à s'autocorriger. Toutefois, cette autocorrection n'est pas spontanée, mais elle s'effectue progressivement dans la démarche réflexive de recherche. Il peut donc arriver qu'elle ne soit pas couronnée de succès (Lipman, 1995, 2006).

\section{Cadre méthodologique}

\section{La stratégie de recherche}

Puisque notre but était de mieux comprendre et de mieux décrire les manifestations de la pensée critique en vue de comprendre son sens (signification et direction) et son utilité dans les pratiques des enseignants, nous avons opté pour une stratégie de recherche de type qualitatif interprétatif 
axée sur l'analyse de cas multiples (Deslauriers et Kérisit, 1997; Huberman et Miles, 1991).

L'approche méthodologique d'étude de cas multiples (multi site ou multicas) nous permet non seulement de mieux respecter les différentes « colorations ou expressions » des manifestations de la pensée critique dans la pratique de chacun des enseignants participant à l'étude, mais en plus comme le mentionnent Huberman et Miles (1991), elle accroît la transférabilité des résultats. Cette approche méthodologique montre, du même coup, que les événements et les processus observés ne sont pas purement idiosyncratiques. Elle augmente la puissance explicative du phénomène de la pensée critique tel qu'il se produit chez les différents participants; ce qui n'est pas le cas d'une approche monosite (Yin, 1994).

\section{Les participantes et les participants et les milieux de l'étude}

Trois enseignants (deux hommes et une femme) d'éducation physique et à la santé de deux écoles secondaires privées de la commune urbaine de Montréal se sont portés volontaires pour participer à l'étude. Tous sont natifs de la province de Québec. Ensemble, ces participants reflétaient des a priori différents (âge, sexe, années d'expérience, culture professionnelle) Le tableau 1 ci-dessous résume le profil des deux enseignants et de l'enseignante.

Tableau I. Portrait de l'enseignante et des enseignants participant à l'étude

\begin{tabular}{|c|c|c|c|}
\hline & Sexe & Âge & Expérience \\
\hline Enseignant A1 & F & 28 ans & 5 ans \\
\hline Enseignant A2 & M & 50 ans & 30 ans \\
\hline Enseignant A3 & M & 35 ans & 8 ans \\
\hline
\end{tabular}

\section{L'instrument de collecte de données et les stratégies de contrôle}

Pour assurer la validité interne, nous avons élaboré notre collecte de données à partir d'un instrument et de six stratégies de contrôle de la précision des réponses. L'instrument est l'entrevue et les stratégies sont : 1) la rencontre pré-classe, 2) la rencontre post-classe, 3) l'observation non participante, 4) l'enregistrement des enseignements, 5) le rappel stimulé et 6) le Feed-back des participantes et des participants.

\section{La rencontre pré-enseignement}

Avant l'observation de chaque enseignement de la participante et des participants, une rencontre préalable avait lieu. Elle avait pour but de recueillir auprès du participant le but de la séance et, si possible, quelques informations sur le fondement des principaux éducatifs prévus. Les principales questions qui leur étaient posées étaient les suivantes : Que vas-tu faire aujourd'hui avec ta classe ? Que veux-tu développer dans ta leçon du jour ? Pourquoi cette 
intention éducative ? Y a-t-il une raison pour laquelle tu prévois faire cet éducatif?

\section{L'observation non participante du chercheur}

Afin de perturber le moins possible le comportement de la participante et des participants, de même que le déroulement de la séance d'enseignement, une observation non participante est effectuée par le chercheur lors de chaque enseignement. Le chercheur se plaçait à une distance raisonnable du plateau d'enseignement afin non seulement de ne pas perturber le déroulement du cours mais aussi d'avoir une vision plus large qui lui permettait d'observer toute la classe.

\section{L'enregistrement des enseignements}

Pour compléter l'observation des actions et comportements de l'enseignante et des enseignants lors du processus enseignementapprentissage, et accroître du même coup la validité interne des données, nous avons ajouté à l'observation non participante du chercheur, l'observation avec support technique (Van der Maren, 1995). Trois enregistrements des enseignements ont été faits pour chaque enseignant et enseignante dans une période de trois semaines, en raison d'un enregistrement par semaine. Tous les enregistrements ont été faits grâce à une caméra VHS fixée à un trépied et pouvant fonctionner à l'aide d'une batterie. Un micro-cravate fut donné aux participants afin d'entendre complètement leurs propos émis en enseignement; mais aussi pour leur permettre de se déplacer sans être gênés.

\section{La rencontre post-enseignement}

La rencontre post-enseignement avait lieu juste après l'observation et l'enregistrement de chaque enseignement des participants. Cette rencontre avait pour intention de recueillir l'évaluation « à chaud » de l'enseignement par l'enseignant ou l'enseignante et, si possible, les pistes d'actions envisagées pour le cours suivant ainsi que les justifications de ces pistes. Les questions suivantes étaient posées à l'enseignant ou à l'enseignante: As-tu été en mesure de réaliser ton intention du jour et les activités prévues ? As-tu fait des adaptations ? Que prévois-tu faire avec ces élèves à la prochaine rencontre ? Pourquoi? Les réponses données par les enseignants participants sont mentionnées sur la fiche de séance du chercheur.

\section{Le rappel stimulé}

La technique du rappel stimulé consiste à revoir avec l'enseignante ou l'enseignant l'enregistrement vidéo de ce qu'il a fait en action et à recueillir simultanément des données sur ce qu'il a vraisemblablement pensé au moment de l'action. Grâce à cette technique, l'enseignante et les enseignants de l'étude 
ont pu reconstituer plus facilement, a posteriori, leurs pensées, leurs décisions et les raisons qui les avaient conduits à agir de telle ou de telle autre façon.

\section{Le feed-back de la participante et des participants}

Le feed-back est une stratégie méthodologique en recherche qualitative qui consiste à faire valider des données par les acteurs sociaux auprès desquels ces dernières ont été recueillies (Boudreau, 2001). Ainsi, après chaque séance de rappel stimulé, le chercheur retranscrivait intégralement, dans un premier temps, le contenu des entrevues semi-dirigées; dans un deuxième temps, cette transcription était remise à l'enseignante et aux enseignants concernés pour vérification. Les enseignants étaient invités à apporter des commentaires au niveau de la forme qu'à celui du fond de la transcription. Ils pouvaient ainsi compléter leur pensée s'ils l'estimaient nécessaire.

\section{L'entrevue semi-dirigée}

Trois entrevues semi-dirigées ont été réalisées auprès de chaque participant et participante à l'étude, soit un total de neuf entrevues. Chacune des entrevues avait lieu lors des séances de rappel stimulé, deux jours au plus tard après l'enregistrement de l'enseignement. Elle avait une durée de soixante à quatre-vingt-dix minutes (60 à 90mn) et était enregistrée, cette fois, sur bande audio. Les entrevues ont été conduites à partir d'une grille composée des éléments liés aux caractéristiques lipmaniennes de la pensée critique. Cela nous permettait de nous assurer d'aborder toutes les composantes importantes de la thématique de la recherche durant l'entretien. Ci-dessous, le guide d'entrevue utilisé lors de l'entretien semi-dirigé.

Tableau II. Guide d'entrevue

\begin{tabular}{|c|c|c|}
\hline \multicolumn{3}{|c|}{ Caractéristique de la pensée critique selon Lipman (1995a, 2006) } \\
\hline Autocorrection & Sensibilité au contexte & Basé sur des critères \\
\hline $\begin{array}{l}\text { - Quelles sont les limites de cette } \\
\text { façon d'enseigner ou de cette } \\
\text { façon de procéder? } \\
\text { - T'arrive-t-il souvent ou parfois } \\
\text { de te corriger à la suite des } \\
\text { prestations motrices observées } \\
\text { chez tes élèves? } \\
\text { - Pourquoi as-tu changé ton } \\
\text { éducatif après quelques minutes } \\
\text { de pratique des élèves? } \\
\text { - Qu'est-ce qui t'a amené à } \\
\text { changer et à proposer les tâches } \\
\text { éducatives non prévues } \\
\text { auparavant dans ta planification? } \\
\text { - T'arrive-t-il souvent ou parfois } \\
\text { de te corriger après une prise de } \\
\text { conscience de ta part suite à un }\end{array}$ & $\begin{array}{l}\text { - Pourquoi as-tu agi de la } \\
\text { sorte ici alors que tantôt } \\
\text { tu avais fait ceci ? } \\
\text { - Est-ce que c'est ainsi } \\
\text { que tu agis toujours? } \\
\text { - En ce moment précis, } \\
\text { pourquoi as-tu utilisé ou } \\
\text { fait ceci ? Y a-t-il une } \\
\text { raison particulière ou } \\
\text { spécifique? } \\
\text { - T'adaptes-tu souvent? }\end{array}$ & $\begin{array}{l}\text { - Sur quels critères } \\
\text { planifies-tu tu } \\
\text { enseignements? } \\
\text { - Sur quels critères } \\
\text { bases-tu tes actes } \\
\text { pédagogiques? } \\
\text { - Pourquoi as-tu fait } \\
\text { cela? Quelles sont tes } \\
\text { raisons? }\end{array}$ \\
\hline
\end{tabular}


éducatif mis en place mais qui est improductif ou qui ne marche pas comme tu l'aurais souhaité ?

\section{La stratégie d'analyse des données}

La stratégie d'analyse du corpus recueillie s'est déroulée sur la base d'une analyse de contenu pour une étude de cas multiples. Elle s'est faite sous la forme d'un processus d'analyse en trois étapes : la codification des données, l'analyse verticale de chaque site (les données issues de chaque enseignant ou enseignante) et l'analyse horizontale (ou transversale) de tous les sites. À la fin de ces étapes, nous avons fait ressortir, de façon inductive, des éléments de définition de la pensée critique dans la pratique des enseignants d'éducation physique et à la santé.

\section{La codification des données recueillies}

La codification du corpus s'est faite à partir d'une grille d'analyse élaborée tenant compte des indicateurs caractérisant la pensée critique tels que relevés par Lipman $(1995,2006)$. Nous avons procédé par un codage fermé adapté à l'acte enseignant pour l'analyse de la forme de la pensée. Dans le cas de la présente étude, les trois principales caractéristiques de la pensée critique définies dans le cadre conceptuel ont servi pour le codage. Toutefois, seuls les éléments de la caractéristique «présence de critères » ont été adaptés pour l'acte enseignant en éducation physique et à la santé.

Une fois la transcription intégrale des neuf (9) entrevues semi-dirigées terminée et validée par les enseignants, nous avons procédé premièrement à un codage de tout le verbatim afin de ressortir les idées générales ou l'essentiel des propos de l'enseignant sans en chercher à le qualifier. Dans une deuxième étape nous avons codé le corpus, de façon fermée et adaptée, en faisant ressortir des unités de sens en lien avec la forme de la pensée critique. En d'autres termes, nous avons fait ressortir des transcriptions des unités informationnelles sur « l'autocorrection », ou sur « la sensibilité au contexte » ou encore sur « la présence de critères ».

\section{L'esquisse d'herméneutique de la pensée critique}

L'esquisse de théorisation s'est faite en deux étapes, au fur et à mesure de l'analyse et du traitement des données. Premièrement au niveau de chaque cas. À ce niveau, nous avons essayé de comprendre le fonctionnement de la pensée critique en enseignement en faisant ressortir les manifestations pratiques des composantes de la pensée critique tout en tentant de les expliquer telles qu'elles apparaissaient. Deuxièmement, après cette démarche interprétative des données de chaque site, nous avons procédé à l'analyse horizontale. À ce niveau, nous avons cherché premièrement à faire ressortir, 
par comparaison des sites, les éléments communs et différents en regard de la forme de la pensée critique. Cette analyse en deux étapes a permis une théorisation de la nature et des manifestations de la pensée critique dans la pratique des enseignantes et des enseignants. Le schéma ci-dessous donne une vue globale de la stratégie d'analyse, en prenant l'exemple de trois enseignants.

\section{Présentation et analyse des résultats}

La présentation et l'analyse des résultats seront faits en fonction des critères de la pensée critique selon Lipman (1995, 2006).

De façon globale, il ressort de l'analyse des résultats relatifs à la forme de la pensée des trois enseignants qu'ils font usage d'une pensée de forme critique lors des interactions avec leurs groupes classes. En effet, dans la pensée de chacun d'eux, il y a la présence des trois caractéristiques lipmaniennes d'une pensée critique. Toutefois, comme l'indiquent la figure III, le degré de présence de chacune de ces caractéristiques est fort variable. Le caractère « sensibilité au contexte » est celui qui est le plus dominant dans la pensée de ces professionnels.

Tableau III. La forme de la pensée des enseignants

\begin{tabular}{|l|c|c|c|}
\hline & $\begin{array}{c}\text { Pensée } \\
\text { autocorrective }\end{array}$ & $\begin{array}{c}\text { Pensée sensible au } \\
\text { contexte }\end{array}$ & $\begin{array}{c}\text { Pensée basée sur } \\
\text { des critères }\end{array}$ \\
\hline Enseignant A1 & 11 & 12 & 5 \\
\hline Enseignant A2 & 9 & 19 & 3 \\
\hline Enseignant A3 & 19 & 22 & 7 \\
\hline Total & 39 & 53 & 15 \\
\hline Pourcentage & $36,4 \%$ & $49,4 \%$ & $11,2 \%$ \\
\hline
\end{tabular}

\section{Le caractère « autocorrection »}

De l'ensemble des unités informationnelles relatives aux caractéristiques de la pensée critique, 36,4\% de celles-ci ont trait à «l'autocorrection». Selon nos analyses, la présence du caractère « autocorrection » dans la pensée de ces enseignants se traduit par l'exercice d'une pensée flexible, non rigide et d'une logique non formelle, et ce, dans l'intention de s'ajuster ou de s'adapter afin de poser des actes pédagogiques viables. Ce qui indique qu'ils admettent non seulement la vulnérabilité de leur pensée, mais qu'ils sont enclins à s'autocorriger ou à reconnaître l'inadéquation de leurs premières pensées, ou encore à relever leurs propres erreurs afin de les corriger pour le bénéfice de l'apprentissage ou du développement de leurs élèves. Ci-dessous quelques illustrations du caractère « autocorrection » chez ces enseignants :

« Aujourd'hui j’ai apporté des images à coller pour aider les filles à trouver des éléments gymniques. Je ne le faisais pas auparavant. 
Mais un cours d'éducation physique est un cours comme les autres. Et tu peux ajouter des choses à chaque fois pour améliorer ton cours. Par exemple, à chaque année, je fais un plan de cours. Mais celui-ci ne reste pas comme cela quand j'enseigne. Je le modifie au fur et à mesure que les comportements des élèves le demandent. Je le retravaille à chaque fois ». (A1e3, p.9)

«Oui, j’ai changé ma préparation sur le champ parce que je ne veux pas que ça soit nécessairement comme je l'ai prévu sur mon plan de cours. Ce n'est pas mon cours, c'est celui des élèves. Là où nous (en tant qu'enseignant), on est plus directif c'est dans le choix des moyens d'apprentissage. Même encore là, avec moi, les élèves peuvent s'exprimer. Parfois ils me disent: monsieur, on ne veut pas de la barre fixe, pourriez-vous nous donner une telle activité ? C'est le cas, ici, du basket-ball que j’ai intégré. J'avais programmé auparavant la gymnastique seulement mais j'ai fait de la place pour le basketball quand j'ai vu que cette première activité était fastidieuse pour les élèves. » (A2e1, p.4)

«Dans la progression de la planification de mon cours, j’avais prévu de supprimer le fait de taper dans les mains pour demander la balle, mais pas dans ce cours-ci. Je trouvais que l'introduire en ce momentci était important même si je ne l'avais pas dit en début du cours et que, non plus, je ne l'avais planifié. Toutefois, pour permettre aux élèves de mieux travailler, de mieux réfléchir dans la réalisation de la tâche assignée, il fallait que je rajoute des contraintes. Les porteurs de balle seront obligés de voir l'ensemble de leurs coéquipiers, analyser leur positionnement avant de faire des passes. Je vais ainsi éliminer les décisions émotives et spontanées » (A3e3, p.6)

Dans le premier extrait de transcription, l'enseignante A1 indique qu'elle a posé une rupture dans sa façon habituelle de diriger ses enseignements. Non seulement elle juge inefficace la stratégie d'enseignement mise en place auparavant, mais elle propose également un ajout en vue d'une amélioration. Ainsi, selon cet extrait, l'enseignante A1 fait une modification en introduisant un nouveau support didactique visuel (« des images à coller ») qui favoriserait l'apprentissage des élèves en gymnastique. L’idée de modification ou de flexibilité de la pensée transparaît autrement dans le deuxième extrait de transcription appartenant à A2. En effet, selon cet enseignant, la centration lors du processus enseignement-apprentissage doit être sur les élèves qui sont, à la fois, bénéficiaire et propriétaire du cours d'éducation physique et à la santé. Cette deuxième fonction des élèves l'oblige 
à incorporer des activités physiques et sportives non planifiées mais suggérées par les élèves. Quant au troisième extrait de transcription, le caractère autocorrection se manifeste chez A3 par l'ajout précipité d'une contrainte non prévue pour la séance du jour. En effet, à la suite de l'analyse des comportements de ses élèves et de la situation d'apprentissage, A3 s'oblige à faire intervenir une nouvelle consigne (une contrainte) qui n'était pas planifiée pour le cours. Pour lui, cette nouvelle consigne non planifiée est nécessaire au développement de la réflexion de ses élèves et à la suppression des comportements émotifs et irréfléchis dans la réalisation des tâches assignées.

En somme, face à des situations qui provoquent, chez ces enseignants, des dissonances cognitives et qui entraînent conséquemment une autoévaluation des postulats sous-tendant leur mise en place, ces professionnels posent de façon consciente des ruptures avec leur pensée initiale. Ce « retournement » conscient et voulu par les enseignants est fait uniquement dans une perspective d'amélioration du processus enseignementapprentissage.

\section{Le caractère « sensibilité au contexte »}

Tel que mentionné précédemment dans le tableau II, des trois caractéristiques de la pensée critique mises de l'avant par Lipman (1995, 2006), le caractère «sensibilité au contexte » est celui qui a été noté le plus souvent dans les extraits de transcription des enseignants. Ce qui suggère que ces éducateurs et éducatrices font preuve d'une pensée adaptable qui reconnaît le caractère singulier de chaque contexte. Selon nos analyses, en effet, les applications des règles de pratique des activités éducatives, les principes pédagogiques d'organisation ou de la gestion du groupe-classe, la gestion de la matière ne s'appliquent pas de façon mécanique et identique à tous les contextes d'enseignement. Dans leurs pratiques pédagogiques, ces enseignants prennent en considération les particularités des divers contextes éducatifs. Ainsi, ils seront sensibles aux caractéristiques psycho-sociocognitives des apprenants, à la nature même de la classe d'éducation physique et à la santé, à la situation de la séance dans la progression pédagogique de la planification annuelle, etc. Ci-dessous, quelques illustrations du caractère « sensibilité au contexte » chez les trois enseignants de l'étude.

« J'ai fait un rappel un peu long ici pour les remettre un petit peu dans le contexte. Parce qu'elles ont juste un cours au début de la semaine et l'autre cours à la fin de la semaine prochaine. Il s'écoule beaucoup de temps, surtout qu'en plus elles sont en secondaire 1. Elles n'ont pas toujours les notions et elles ne se souviennent pas nécessairement ce qu'elles ont réalisé la semaine d'avant si on ne les stimule pas » (A1e1, p.9) 
« Non! Un enseignant pour moi n'est pas un applicationniste. Je verrai ceci dans un cadre élitiste car là, il faut qu'on soit beaucoup plus perfectionniste. Ici, ce n'est pas du tout dans ce contexte. Nous avons affaire à une formation scolaire que j'appelle de base. J'ai une classe hétérogène, un amalgame d'individus avec des compétences différentes, n'ayant pas tous les mêmes goûts, ni les mêmes aptitudes. Dans le cadre élitiste, j'ai un cadre très rigide où je sortirai moins du cadre prévu car je vise une performance, mais pas ici. C'est ce qui me fait agir ainsi » (A2e3, p.12)

«J'ai demandé aux élèves de faire un certain nombre de passes avant de pouvoir tirer au panier. Ce qui ralentirait le jeu et permettrait ainsi aux élèves en difficulté d'avoir le temps de mieux voir le jeu, de même que de recevoir des passes. En fait, souvent puisqu'ils ont de la difficulté à dribbler, ces élèves moins habiles ne reçoivent pas de passes de leurs pairs plus habiles. Il faut donc faire en sorte qu'ils aient la chance d'avoir la balle » $(\mathrm{A} 3 \mathrm{e} 2, \mathrm{p} .5)$

Nous avons ici trois extraits de transcriptions qui illustrent quelques manifestations de la sensibilité au contexte de la pensée des trois enseignants. Dans le premier extrait, l'enseignante A1 justifie la longueur de son rappel pédagogique par la prise en considération de deux particularités du contexte éducatif : la longueur de l'intervalle entre deux cours d'éducation physique et à la santé et, les caractéristiques cognitives des élèves (une connaissance limitée et un fréquent oubli des réalisations faites caractérisant les élèves de secondaire 1). Il est impossible, pour elle, d'être indifférente à de telles réalités de l'environnement pédagogique si elle désire voir les élèves apprendre. Dans le deuxième extrait provenant de l'enseignant $\mathrm{A} 2$, la nature et la mission de l'éducation physique et à la santé sont des facteurs qui orientent son agir professionnel. L'éducation physique et à la santé est, pour lui, une « éducation de base » et non une « formation élitiste ». Elle doit permettre à tous les élèves de sa classe, classe fondamentalement hétérogène, de faire des apprentissages. Son agir professionnel est donc situé en regard à l'essence même du cours d'éducation physique et à la santé. Le troisième extrait de transcription fait état de la sensibilité de l'enseignant A3 à l'égard des élèves en difficulté de sa classe. En effet, la pédagogie plus inclusive utilisée s'expliquerait par un souci de permettre aux élèves moins habiles, sur le plan technique, d'avoir accès aux balles et ainsi, avoir des occasions d'apprentissage. Ces élèves en difficultés, selon les propos de A3, justifieraient l'émission de la consigne de faire plusieurs passes à ses coéquipiers avant de tirer au panier. Ainsi la pratique pédagogique de cet enseignant tient compte du développement des habiletés techniques de certains élèves. 
Nous nous apercevons au travers ces trois extraits ci-dessus mentionnés que les pratiques pédagogiques de ces enseignants sont motivées par une conscience et une prise en considération de différents facteurs spécifiques à chaque environnement éducatif. Cette sensibilité au contexte, un des critères de la pensée critique selon Lipman (1995, 2006), est orientée vers l'apprentissage et le développement des élèves.

\section{Le caractère « présence de critères »}

Telles que mentionnées dans le tableau II, les données relatives aux rationnels sous-tendant l'agir professionnel des enseignants de notre étude mentionnent la présence de critères. L'analyse horizontale des unités de sens relatives à cette troisième caractéristique de la pensée critique révèle que, bien que les critères soient «les raisons» qui justifient leurs pratiques pédagogiques, ils sont de double nature. Ainsi, les critères peuvent être soit de nature technique (la manière de dribbler, de faire des passes; la qualité du geste technique, la qualité de la reproduction, etc.), soit de nature socio-éthique (vivre la socialisation dans un groupe pédagogique, respecter les pairs, respecter les consignes de fonctionnement, respecter les consignes de sécurité, être équitable, etc.). Les trois extraits ci-dessous donnent quelques illustrations de la présence de critères dans la pensée critique des enseignants.

«Il y a certaines filles dans les groupes qui ont une certaine connaissance de la gymnastique, donc si elles n'ont pas les photos, ces filles-là vont souvent montrer l'exemple. Elles montrent aux autres membres du groupe le mouvement et elles se fient là-dessus. Alors les standards que je détermine ne sont pas en vérité des standards techniques de la gymnastique sportive comme tels, mais il faut que ça ressemble un petit peu au mouvement. Et en même temps, elles apprennent des mots de vocabulaires. » (A1e3, p.4)

«Quand je dis aux élèves que ça fonctionne bien, c'est en fonction des critères et consignes établis au début. On s'est dit, par exemple au water-polo, qu'on détermine des tâches : tu devras te retrouver sur le jeu avec cinq personnes ayant des positions bien déterminées et en respectant certaines règles. Moi, je vois que c'est respecté. On a le bon nombre sur le jeu, on a déterminé des tâches, on voit très bien la structure du jeu. (...) C'est toujours en regard aux objectifs qu'on s'est fixé. » (A2e1, p11)

«Le critère qui m'a permis de dire «c'est beau » à la prestation motrice de cet élève est qu'il a fait un changement de direction et a sorti sa main pour recevoir le ballon. C'est ce que je voulais que ces 
élèves fassent dans cet éducatif. Lui, il a été capable de faire un changement de direction et de sortir la main » $(\mathrm{A} 3 \mathrm{e} 2, \mathrm{p} .8)$

Dans le premier extrait de transcription provenant de l'enseignante A1, le critère mis en évidence par l'enseignante ici est de nature socio-éthique : le respect de la sécurité. Pour A1, en fait, il n’y a pas « un seul chemin » désigné que doivent emprunter les élèves lorsqu'elles sortent le matériel didactique de la remise. Le critère implicite de l'enseignante est celui du respect de la sécurité par les élèves. Tout comportement des élèves respectant ce principe, assez large, est acceptable par elle. Pour l'enseignant A2, à contrario, le critère mis de l'avant est de nature technique en lien avec la production d'une stratégie technique collective. L'enseignant ne dicte pas exactement ce que les élèves doivent faire mais il s'attend à ce qu'ils adoptent un comportement collectif en arrimage avec les objectifs poursuivis dans la séance. Quant à l'extrait de A3, le critère souligné est de nature technique: la production d'un comportement moteur pertinent en rapport avec la technique du démarquage et d'appel de balle au basket-ball. L'enseignant va donc se baser sur la qualité de la reproduction de ce geste technique pour comparer ses élèves.

Notons que toutes ces raisons qui constituent des critères de la pensée en action des enseignants n'ont pas trait à des valeurs mais à des aspects techniques de l'enseignement et de l'apprentissage. L'existence de ces critères dans l'agir professionnel de ces enseignants suggère que le mode de pensée déployé est de type critique dans le sens où il a recours à des critères pour comparer leurs élèves.

\section{Discussion}

Quelle compréhension peut-on dégager du sens de la pensée critique à partir de ses manifestations dans le contexte psychomoteur ? Pour répondre à cette question, nous procéderons à une discussion des résultats obtenus au niveau de chacune des caractéristiques de la pensée critique selon Lipman (1991, 2006).

\section{Au niveau du caractère « autocorrection » de la pensée critique}

L'analyse des données relatives au caractère «autocorrection » indique que ces enseignants font preuve de remise en cause de leur pensée initiale sous-tendant leurs pratiques pédagogiques. Ce souci d'autoquestionnement revêt une importance particulière dans et pour l'agir professionnel de ces enseignants. En effet, ce « retournement pédagogique » est non seulement nécessaire au développement de leur professionnalité (Schön, 1987, 1994), mais aussi à la construction d'un agir professionnel pertinent. À l'instar des travaux de Daniel (1998b) et de ceux de Lipman (1995, 2006), l'analyse des données laisse transparaître que ces enseignants 
adoptent non seulement une posture de recherche, mais également font preuve d'ouverture face à leurs propres perspectives, ainsi qu'à leur processus éducatif. De plus, la lecture des manifestations ayant trait à ce caractère indique que ces enseignants réfléchissent dans l'action (Schön, 1987, 1994) et sont, non seulement animés d'une recherche d'un certain sens d'adéquation ou de viabilité de leurs actes pédagogiques (Lipman, 1995), mais ils sont aussi dans une attitude d'écoute. Ce qui les distancie d'une certaine rigidité intellectuelle pour les rapprocher d'une liberté intellectuelle (Pallascio, Daniel et Lafortune, 2004).

\section{Au niveau du caractère « sensibilité au contexte » de la pensée critique...}

Le déploiement de ce caractère par ces enseignants, selon nos analyses, est dans le but prioritaire de reconstruire le problème que pose une situation éducative inconfortable à laquelle ils se trouvent confronté. Or, cette reconstruction passe inévitablement par la compréhension du contexte éducatif. Ainsi, à l'opposé des croyances de la conception positiviste et techniciste, les situations de dissonance cognitive rencontrées, par les enseignants de notre étude, ne se présentent pas à eux comme des situations simples, non contextualisables et non problématisables. Il ne semble pas exister de solutions préconçues à ces situations problématiques. Cette enseignante et ces enseignants sentent alors l'obligation de construire le problème en passant par la compréhension des éléments indéterminés de la situation et du contexte en vue d'apporter une solution cohérente à la situation problématique.

Notons, par ailleurs, que s'il est possible de postuler que la présence du caractère « autocorrection » dans la pensée de ces professionnels révèle une certaine ouverture d'esprit à l'égard de leurs limites, donc une ouverture à euxmêmes, le caractère «sensibilité au contexte » suggère, quant à lui, une orientation de leur pensée vers l'extérieur, donc vers autrui. En effet, la prise en compte des caractéristiques de l'environnement physique (caractéristique du gymnase, situation de la séance, le temps alloué à la séance, etc.), de l'essence de l'éducation physique et à la santé ( une discipline scolaire, une formation de base pour tous les élèves, une pratique d'activités physiques pour tous, donc non élitiste, etc.) et surtout des caractéristiques des apprenants (leur degré de familiarité avec l'activité physique utilisée comme moyen d'éducation, leurs caractéristiques psychosociomotrices, leurs développements cognitifs, etc.) dans la conception de leurs pratiques éducatives souligne leur souci de la qualité de leurs actes d'enseignement. Or, s'ils enseignent c'est pour voir leurs élèves apprendre. Et par ce fait même, la présence de ce caractère indique leur souci de l'apprentissage des élèves. À ce niveau, nous pourrions inférer que cette perspective sociocentrique de la pensée critique, via la forte présence du caractère de la sensibilité au contexte, 
suggère que cette enseignante et ces enseignants souscrivent dans une perspective de déploiement d'une pensée critique au sens fort (Paul, 1992) ${ }^{1}$.

\section{Lecture des manifestations du caractère « basé sur des critères » de la pensée critique}

Dans l'agir professionnel de l'enseignante et des enseignants de notre étude, les données analysées révèlent la présence de critères ou de raisons sous-jacentes aux pratiques éducatives de l'enseignante et des enseignants. Toutefois, chez tous ces enseignants, la présence de ce troisième caractère de la pensée critique est relativement faible. Or, si pour Paul (1992) la pensée critique au sens fort s'exprime dans l'altruisme, pour Lipman $(1991,1995)$ la présence de ce critère est fondamentale à une pensée critique au sens fort. Lipman affirme que « la pensée critique est une pensée qui, à la fois, utilise des critères et existe par son recours à des critères » (Lipman, 1995, p. 146). Dès lors que ce caractère se fait rare chez ces enseignants, il est possible de s'interroger sur la robustesse de leur pensée de forme critique. En effet, ce caractère éviterait de qualifier le jugement ou les décisions de ces professionnels de décision prise de façon intuitive et gratuite. Les critères sont donc essentiels à la pensée critique car, selon Lipman (1995, 2006), ils lui donnent du poids.

Par ailleurs, si Lipman (1995) conçoit que «la pensée critique constitue une responsabilité cognitive pour l'enseignant, dans le sens où celuici doit ressentir l'obligation de fournir ouvertement les critères qui sont les siens à des opinions et alléger le développement de l'autonomie intellectuelle des élèves » (p. 147-148), force est de constater que les résultats de notre étude révèle que les jugements de cette enseignante et de ces enseignants ne sont basés que sur peu de critères clairement identifiables, ce qui affaiblit la nature critique, même au niveau de la forme, de leur pensée. Cette rareté du caractère «basé sur des critères » pourrait laisser entrevoir que ces professionnels de l'enseignement agiraient de façon « quasi réflexive » (versus « réflexive ») selon King et Kitchner (1994), lesquelles s'inspirent de l'épistémologie de Dewey (1933). Ainsi, ils ne se préoccuperaient pas tant du pourquoi de leur jugement lors de l'interaction avec leurs élèves, mais surtout du « comment ».

1 Nous voulons rappeler ici, la conception Paulinienne de la pensée critique. En effet, pour Paul (1992), la pensée critique est une pensée disciplinée qui se guide elle-même et qui représente la perfection de la pensée appropriée à un certain mode ou domaine de la pensée. Il distingue deux formes de pensée critique : 1) la pensée critique au sens faible, qui est égocentrique et sert donc les intérêts d'un individu ou d'un groupe particulier, 2) la pensée critique au sens fort, qui est sociocentrique et qui prend en compte les intérêts d'une diversité de personnes et qui a, de plus, intégré aux habiletés de pensée critique les valeurs de vérité, de rationalité, d'autonomie et de connaissance personnelle. 
Si nous nous arrêtons à la nature des rares critères présents qui soustendent les jugements de cette enseignante et de ces enseignants lors du processus enseignement-apprentissage, nous constatons qu'elle est double. L'analyse des données révèlent, en effet, que certains sont de nature technique, donc des faits (gestes, habiletés motrices à produire ou à reproduire, qualité des actions motrices, etc.) et d'autres, de nature socioéthique, donc des valeurs (socialisation, mixité du groupe, équité, etc.). Toutefois, à l'analyse des données, quelle que soit la nature des critères, les fonctions assumées sont les mêmes. Tous les critères émis servaient de base de comparaison ou d'évaluation des prestations motrices des élèves.

Bref, que pouvons-nous conclure sur la forme de la pensée critique présente dans la pratique de l'enseignante et des enseignants de cette étude ? D'abord, lorsque nous considérons la forme de la pensée critique dans sa globalité, nous constatons la présence de toutes les caractéristiques de la pensée critique telles qu'identifiées par Lipman. Toutefois, ces caractéristiques sont d'importance inégale dans la pratique de cette enseignante et de ces enseignants. La pensée critique, dans la pratique, est fortement dominée par le caractère «sensibilité au contexte », offre une présence acceptable du caractère «autocorrectif», mais une faible représentation du caractère même qui détermine l'aspect critique de la pensée critique selon Lipman, c'est-à-dire la présence de critères sous-tendant le jugement de cette éducatrice et de ces éducateurs physiques. Les critères émis par les trois enseignants ne sont pas tous de nature conceptuelle (donc reposant sur des valeurs), mais ils sont surtout de nature technique, c'est-à-dire reposant sur des faits et des gestes.

\section{Conclusion}

La présente étude a tenté, à partir des manifestations de la forme de la pensée critique déployée par trois enseignants d'éducation physique et à la santé, de présenter une compréhension de ce mode de pensée. Selon l'analyse des données recueillies, les caractéristiques lipmaniennes de la pensée critique se présentent comme des éléments-leviers sur lesquels les enseignants d'éducation physique s'appuient pour tenter de donner un sens à leur intervention éducative. Ainsi, les enseignants font appel aux caractéristiques du contexte éducatif (c'est-à-dire qu'ils sont « sensibles au contexte ») pour les aider à problématiser les situations éducatives problématiques; ils font appel au caractère «autocorrection » de la pensée critique pour les rendre conscients de leur vulnérabilité et pour s'inscrire dans une perspective d'amélioration de leurs actes pédagogiques. Quand ils s'appuient sur les « critères » ou des «principes et raisons », ils veulent rendre des décisions robustes, c'est-à-dire non prises de façon intuitive et gratuite. La pensée critique déployée par ces enseignants en exercice se présente essentiellement 
comme un processus cognitif de résolution de situations pédagogiques problématiques pour le bénéfice des élèves.

\section{References:}

1. Bennett, G., et Green, F.P. (2001). Student Learning in Online Environment : No Significant Difference? Quest, 53(1), 1-13.

2. Boudreau, P. (2001). Que se passe-t-il dans un stage réussi ? Revue des Sciences de l'Éducation, 27(1), 65-84.

3. Cleland, F.D., Helion, J., et Fry, F. (1999). Modifying Teacher Behaviors to Promote Critical Thinking in K-12 Physical Education. Journal of Teaching in Physical Education, 18, 199-215.

4. Daniel, M.-F. (1998a). La pratique philosophique et le développement de la pensée critique chez les futurs enseignants en éducation physique. Dans L. Lafortune, P. Mongeau et R, Pallascio, Métacognition et compétences réflexives (p.285-311). Québec : Les Éditions Logiques.

5. Daniel, M.-F. (1998b). La philosophie et les enfants. Montréal : Logiques.

6. Daniel, M.-F. (2001). Philosophical dialogue among peers: a study of manifestations of critical thinking in preservice teachers. Advances in Health Sciences Education, 6, 49-67.

7. Daniel, M.-F., Bergman-Drewe, S. (1998). Higher order thinking, philosophy and teacher formation in physical education. Quest, 50(1), 33-59.

8. Deslauriers, J.-P. et Kérisit, M. (1997). Le devis de recherche qualitative. Dans J. Poupart, J.-P. Deslauriers, L.-H. Groulx, A. Laperrière, R. Mayer et A.P. Pires, La recherche qualitative. Enjeux épistémologiques et méthodologiques (p. 85-111). Gaëtan Morin Éditeur.

9. Dewey, J. (1933). How We Think Boston: D.C. Heath.

10. Doherty, J. (2009). Critical Thinking in Physical Education. Teaching and Development of Critical Thinking in Physical Education. VDM Verlag: Saarbrücken.

11. Durand, M. (1998). L'enseignement en milieu scolaire ( $2^{\mathrm{e}}$ édition). Paris : PUF.

12. Ennis, R. H. (1991). Critical thinking: A streamlined conception. Teaching Philosophy, 14(1), 5-25.

13. Gaskins, I. W (1994). Classroom applications of cognitive science. Teaching poor readers how to learn, think, and problem solve. Dans K. McGilly (dir.), Classroom Lessons (p. 129-154). Cambridge, MA : MIT Press. 
14. Howe, E. R. (2004). Canadian and Japanese teachers' conceptions of critical thinking: A comparative study. Teachers and Teaching: Theory and Practice, 10(5), 505-525.

15. Huberman, A.M. et Miles, M.B. (1991). Analyse des données qualitatives: Recueil des nouvelles méthodes. Bruxelles : De Boeck.

16. King, P.M. et Kitchner, K.S. (1994). Developing Reflective Judgment. San Francisco : Jossey-Bass.

17. Knight, C.L.H. (1992). Teaching critical thinking in the social sciences. New Directions for Community College, 73, 63-73.

18. Kpazaï, G. (2018). (2018). La pensée critique expliquée par des didacticiennes et des didacticiens de l'enseignement supérieur (2e édition). Montréal (Québec) : Les Éditions JFD Inc.

19. Kpazaï, G. (2015). Pensée critique et innovations dans la formation universitaire. Côte Saint-Luc, Montréal (Québec): Éditions Peisaj.

20. Kpazaï, G. (2014). La présence de la pensée critique chez les enseignants d'éducation physique et sportive : un miroir à la compétence professionnelle en éducation physique. Revue des Sciences et Pratiques des Activités Physiques Sportives et Artistiques, 6 (2), 1-16.

21. Kpazaï, G. (2013). Étude de la pensée critique en éducation physique et santé. Les manifestations de la pensée critique chez les enseignants d'éducation physique et santé. Saarbrücken: Presses Académiques Francophones.

22. Kpazaï, G., Daniel, M.-F., \& Attiklemé, K. (2015). A Pedagogical Analysis of Critical Thinking deployed by Health and Physical Education Teachers at the Secondary School Level. International Journal of Kinesiology \& Sports Science, 3 (3), 1-12. doi:10.7575/aiac.ijkss.v.3n.3p.1

23. Kpazaï, G., Daniel, M.-F. \& Attiklemé, K. (2011). Manifestations of Critical Thinking in Health and Physical Education Teachers: An Examination of Three Case Studies. PHENex Journal, Vol 3 (2), 1-15

24. Lafortune, L. et Robertson, A. (2004). Métacognition et pensée critique. Dans R. Pallascio, M.-F. Daniel et L. Lafortune (dir.), Pensée et réflexivité : Théories et pratiques (p. 107-128). Sainte-Foy : Presses de l'Université du Québec.

25. Lipman, M. (1995). À l'école de la pensée. (Traduit de l'anglais par Nicole Decostre). De Boeck Université.

26. Lipman, M. (2006). À l'école de la pensée (Édition revue et ajoutée). De Boeck Université.

27. McBride, R.E. (1999). If you Structure it, they will learn... Critical thinking in physical education classes. Clearing House, 72(4), $217-$ 220. 
28. McBride, R.E, et Xiang, P. (2004). Thoughtful Decision Making in Physical Education: A Modest Proposal. Quest, 56, 337-354.

29. Ministère de l'éducation de la Colombie Britannique (2002). Graduation requirements review: history of British Columbia school revisions and major themes. Site internet : http://www.bced.gov.bc.ca/grad_req_rev/research/history.htm

30. Ministère de l'Éducation de la Nouvelle Zélande (2005). Proposed Key Competencies and Health And Physical Education in the New Zealand Curriculum. Accessed from https://www.researchgate.net/profile/Lisette_Burrows/publication/23 9581366_Proposed_Key_Competencies_and_Health_and_Physical_ Education_in_the_New_Zealand_Curriculum/links/0c9605335de40e 352b000000/Proposed-Key-Competencies-and-Health-and-PhysicalEducation-in-the-New-Zealand-Curriculum.pdf

31. Ministère de l'Éducation de l'Ontario. (2015). Compétences du 2le siècle : Document de réflexion. Phase 1 : Définir les compétences du 21e siècle pour l'Ontario. Repéré à http://pedagogienumeriqueenaction.cforp.ca/wp-content/ uploads/2016/02/Ontario-21st-century-competencies-foundationFINAL-FR_AODA_EDUGAINS_Feb-19_16.pdf

32. Ministère de l'Éducation du Québec (2001a). Programme de formation de l'école québécoise: Éducation préscolaire, enseignement primaire. Québec : Gouvernement du Québec.

33. Ministère de l'Éducation du Québec (2001b). La formation à l'enseignement. Les orientations, les compétences professionnelles. Québec : Gouvernement du Québec.

34. Ministère de l'Éducation du Québec (2003). Programme de formation de l'école québécoise: Enseignement secondaire, premier cycle. Québec : Gouvernement du Québec.

35. Ministère de l'Enseignement Primaire et Secondaire (2006). Programmes d'études. Éducation physique et Sportive, version révisée. République du Bénin.

36. Newman, F.M. (1990). Higher order thinking in teaching social studies: A rationale for the assessment of classroom thoughtfulness. Journal of Curriculum Studies, 22(19), 41-56.

37. Pallascio, R.; Daniel, M.-F et L. Lafortune (dir.) (2004). Pensée et Réflexivité : Théories et pratiques. Sainte-Foy : Presses de l'Université du Québec.

38. Paul, R. (1992). Critical thinking: What, Why and How. New Directions for community College, 77, 3-24.

39. Roy, A. (2005). Manifestations d'une pensée complexe chez un groupe d'étudiants-maîtres $d u$ primaire à l'occasion d'un cours de 
mathématique présenté selon une approche philosophique. Thèse de doctorat non publié. Université du Québec à Montréal, Faculté des sciences de l'éducation.

40. Schön, D.A. (1987). Educating the Reflective Practioner. San Francisco : Jossey Bass.

41. Schön, D.A. (1994). Le practicien réflexif. À la recherche du savoir caché dans l'agir professionnel Traduit par J. Heynemand et D. Gagnon. Montréal : Les Éditions Logiques.

42. Sebren, M.A. (1995). Preservice teachers'reflections and knowledge development. Journal of Teaching in Physical Education, 14, 262-283.

43. Shavelson, R. J.(1973), What is basic teaching skill? Journal of Teacher Education, 14, 144-151.

44. Smyth, J. (1984). Teacher as collaborators in clinical supervision: cooperative learning about teaching. Teacher Education, 24, 60-68.

45. Taylor, P.H. (1970). How Teachers Plan their Courses, London: National Foundation for Educational Research in England and Wales.

46. Trudel, P., Haughian, H. et Gilbert, W. (1996). L'utilisation de la technique du rappel stimulé pour mieux comprendre le processus d'intervention de l'entraîneur en sport. Revue des sciences de l'éducation, 22(3), 503-522.

47. Tsangaridou, N. et Siedentop, D. (1995). Reflective Teaching: A literature Review, Quest, 47, 212-237.

48. Van der Maren, J-M. (1995). Méthodes de recherche pour l'éducation. Les Presses de l'Université de Montréal et De Boeck Université.

49. Yin, R.K. (1994). Case Study Research: Design and Methods $\left(2^{\text {nd }}\right.$ édition). Newbury Park : CA: Sage. 